\title{
Broccoli-Derived Sulforaphane and Chemoprevention of Prostate Cancer: From Bench to Bedside
}

\author{
Ali I. Amjad ${ }^{1,2}$ - Rahul A. Parikh ${ }^{1,2}$ - Leonard J. Appleman ${ }^{1,2}$. \\ Eun-Ryeong Hahm ${ }^{2,3} \cdot$ Kamayani Singh ${ }^{2} \cdot$ Shivendra V. Singh ${ }^{2,3}$
}

Published online: 16 April 2015

(C) Springer International Publishing AG 2015

\begin{abstract}
Sulforaphane (SFN) is a metabolic byproduct of cruciferous vegetables and is the biologically active phytochemical found in high concentrations in broccoli. It has been studied extensively for its anticancer efficacy and the underlying mechanisms using cell culture and preclinical models. The immediate precursor of SFN is glucoraphanin, a glucosinolate which requires metabolic conversion to SFN. SFN and other notable isothiocyanates (ITCs), including phenethyl isothiocyanate and benzyl isothiocyanate found in various cruciferous vegetables, have also been implicated to have a chemopreventive role for breast, colon, and prostate cancer. In vitro and in vivo anticancer activity of this class of compounds summarizing the past two decades of basic science research has previously been reviewed by us and the others. The present review aims to focus specifically on SFN and its chemopreventive and antineoplastic activity against prostate cancer. Particular emphasis in this communication is placed on the current status of clinical research and prospects for future clinical trials with the overall objective to better understand the clinical utility of this promising chemopreventive nutraceutical in the context of mechanisms of prostate carcinogenesis.
\end{abstract}

This article is part of the Topical Collection on Cancer Chemoprevention

Ali I. Amjad

amjadai@upmc.edu

1 Division of Hematology/Oncology, Department of Medicine, University of Pittsburgh School of Medicine, Pittsburgh, PA, USA

2 University of Pittsburgh Cancer Institute, University of Pittsburgh Medical Center, Pittsburgh, PA, USA

3 Department of Pharmacology and Chemical Biology, University of Pittsburgh School of Medicine, Pittsburgh, PA, USA
Keywords Sulforaphane $\cdot$ Prostate cancer $\cdot$ Chemoprevention

$\begin{array}{ll}\text { Abbreviations } \\ \text { AR } & \text { Androgen receptor } \\ \text { CDK } & \text { Cyclin-dependent kinase } \\ \text { DNMT } & \text { DNA methyltransferase } \\ \text { GST } & \text { Glutathione } S \text {-transferase } \\ \text { HDAC } & \text { Histone deacetylase } \\ \text { ITC } & \text { Isothiocyanate } \\ \text { NAC } & \text { N-acetyl cysteine } \\ \text { NQO1 } & \text { NAD(P)H dehydrogenase, quinone 1 } \\ \text { NF-KB } & \text { Nuclear factor-kappaB } \\ \text { PIN } & \text { Prostatic intraepithelial neoplasia } \\ \text { PI3K } & \text { Phosphatidylinositide 3-kinase } \\ \text { PSA } & \text { Prostate specific antigen } \\ \text { PTEN } & \text { Phosphatase and tensin homolog } \\ \text { ROS } & \text { Reactive oxygen species } \\ \text { SFN } & \text { Sulforaphane } \\ \text { TRAMP } & \text { Transgenic adenocarcinoma of mouse prostate }\end{array}$

\section{Introduction}

Prostate cancer accounts for approximately $28 \%$ of newly diagnosed cancers in the USA and is the second leading cause of cancer-related deaths in men in the Western world. According to the 2014 US estimates, 233,000 new patients will be diagnosed with prostate cancer, and 29,480 will die from the disease [1].

Dietary intake of cruciferous vegetables is associated with a reduction in prostate cancer risk in epidemiological studies. In the year 2000, Kolonel et al. published a comprehensive case-control study of 3237 men, observing a $39 \%$ reduction in prostate cancer risk between subjects with the highest and 
lowest quintile of cruciferous vegetable intake. Interestingly, this inverse relationship of prostate cancer risk with cruciferous vegetable intake was also noted for the Chinese and Japanese ethnic groups but not in the Caucasian or African-American subgroups [2]. In another study from the same year, Cohen et al. noted that increasing cruciferous vegetable intake from one serving per week to more than three servings per week was associated with a $41 \%$ reduction in risk for development of prostate cancer. This population was predominantly made up of Caucasian and African-American participants [3]. A possible reason for discrepancy between the two studies could be the type of vegetables classified as cruciferous. While Cohen et al. had a very narrow definition, the study by Kolonel et al. included green mustard cabbage, head cabbage, mustard greens, pak choy, red cabbage, and turnip greens. Over the years, there have been many other epidemiological studies studying this association. A recent meta-analysis from the year 2012 combined seven cohort and six population-based casecontrol studies showing that higher intake of cruciferous vegetables was associated with a decreased risk of prostate cancer $(\mathrm{RR}=0.90,95 \% \mathrm{CI} 0.85-0.96)$ [4••]. A cohort study from Germany ( $n=11,405$ men) demonstrated the strongest association of aliphatic glucosinolate content of food and decreased incidence of prostate cancer, thus strengthening the argument for this inverse association $[5 \cdot \bullet]$.

Anticancer effect of cruciferous vegetables is attributed to isothiocyanate (ITC) class of chemicals characterized by the presence of $\mathrm{N}=\mathrm{C}=\mathrm{S}$ group. The cruciferous vegetables differ with respect to the level of ITCs. For example, broccoli is a rich source of sulforaphane (SFN), whereas phenethyl isothiocyanate is predominant in watercress. Other commonly studied ITCs include benzyl isothiocyanate and allyl isothiocyanate. The topic of cancer chemoprevention with dietary ITCs is covered elsewhere $[6 \bullet \bullet]$. This article focuses on preclinical and clinical evidence supporting the role of SFN in prostate cancer chemoprevention. Metabolism of glucoraphanin to SFN and its metabolites is summarized in Fig. 1. In broccoli and its sprouts, SFN exists in its relatively inert precursor form called glucoraphanin, which is a glucosinolate. Glucoraphanin is converted to active SFN by myrosinase (Fig. 1), an enzyme that coexists but is physically segregated in intact plant cells. Myrosinase is not present in mammalian cells but is found in gastrointestinal microflora in humans. There is some evidence for degradation of glucoraphanin content and inactivation of plant myrosinase depending on conditions of storage, preparation, and mechanism of cooking [7]. Therefore, in theory, conversion of any glucoraphanin to SFN in cooked broccoli may depend on gut microflora [8]. After absorption of SFN, it is metabolized by the mercapturic pathway into sequential metabolites, collectively called dithiocarbamates. These metabolites include glutathione conjugate of SFN (SFNGSH), cysteinylglycine conjugate of SFN (SFN-Cys-Gly), cysteine conjugate of SFN (SFN-Cys), and $N$-acetyl cysteine conjugate of SFN (SFN-NAC).

\section{Mechanisms of Chemoprevention}

Over the years, there has been extensive literature demonstrating preclinical efficacy of SFN against a wide variety of malignancies. Preclinical studies have utilized a racemic synthetic analogue of SFN (D,L-sulforaphane). We summarize here the potential cellular mechanisms pertinent to chemoprevention in prostate cancer. It is to be noted that all these mechanisms have some degree of interaction to synergistically afford chemoprevention (Fig. 2).

\section{Defense Against Carcinogens}

Early research was focused on the effect of SFN on carcinogen detoxification by virtue of its ability to induce glutathione $S$-transferases (GST). This leads to increased conjugation of electrophilic metabolites of environmental carcinogens with glutathione. The biological activity of SFN was first recognized in the early 1990 s $[9,10 \bullet]$. A major mechanism by which it was deemed to be "chemopreventive" was the inhibition of carcinogen-activating enzymes (cytochrome P450) and induction of carcinogen-detoxifying enzymes (phase 2 enzymes including heme oxygenase- 1 and $\mathrm{NAD}(\mathrm{P}) \mathrm{H}$ dehydrogenase, quinone 1 (NQO1)) [11]. It is now well-understood that induction of phase 2 enzymes is mediated by the Keap1-Nrf2-antioxidant response element axis [12]. SFN binds with cytosolic Keap1 (kelchlike ECH-associated protein 1) and releases the transcription factor Nrf2 (nuclear factor, erythroid 2-like 2) to translocate into the nucleus where it binds to the antioxidant response element. This cascade of events leads to regulation of genes involved in cellular defense and survival $[12,13]$. SFN produces robust and sustained transcriptional induction of NQO1 gene expression that was accompanied by similar increases in NQO1 enzymatic activity in human prostate cells in vitro [14].

The most common molecular genetic change in prostate cancer involves epigenetic silencing of the gene encoding GSTP1, a critical isoenzyme of carcinogen defense belonging to the GST family. This change was noted in a majority ( $>90 \%$ ) of prostate adenocarcinomas and nearly $70 \%$ of high-grade prostatic intraepithelial neoplasia (PIN) [15]. It is possible that SFN restores normal GST function and thus helps fight carcinogen-mediated damage during prostate cancer initiation. 


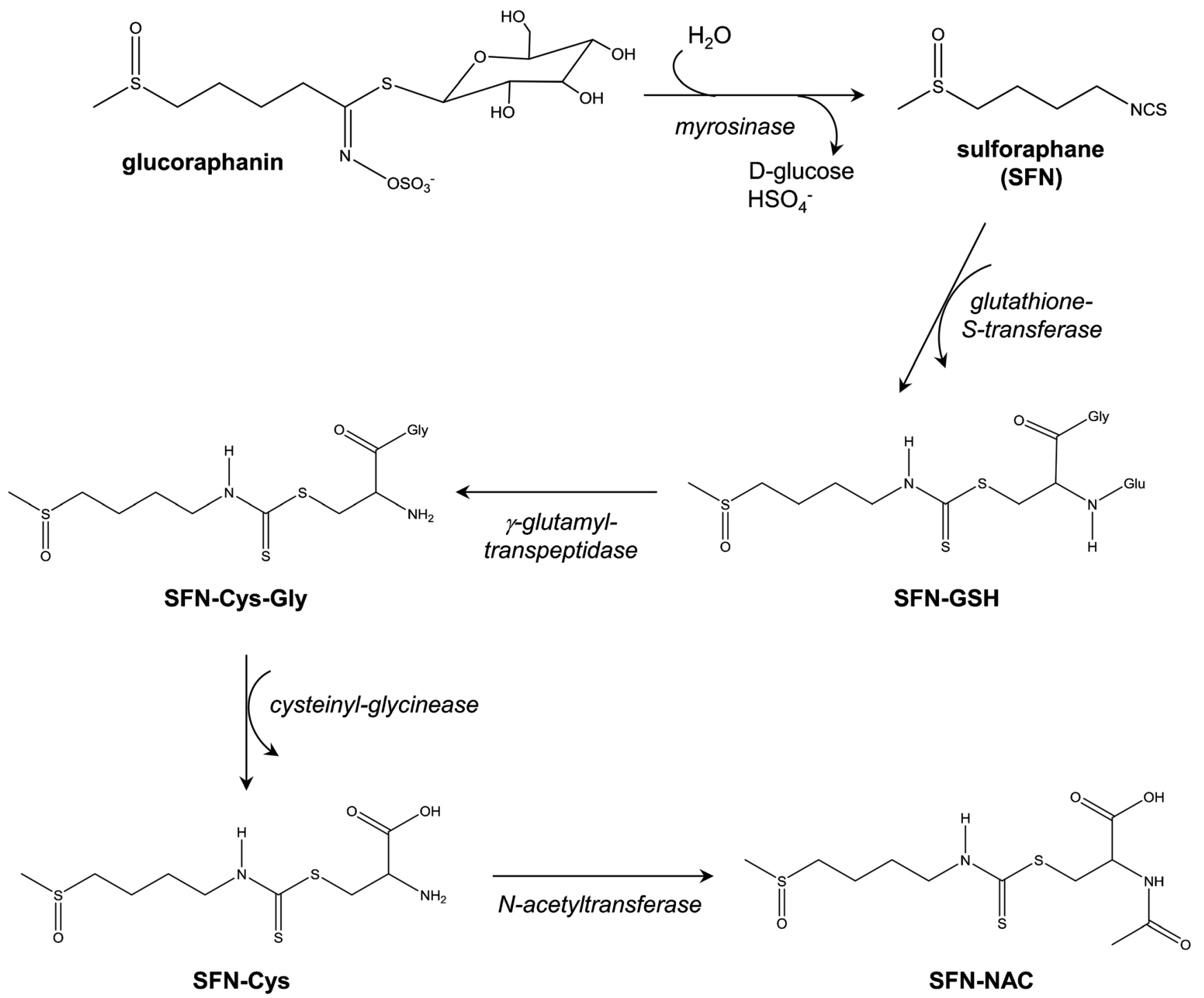

Fig. 1 Schematic of metabolic conversion of glucoraphanin to SFN and its metabolites, including glutathione conjugate of SFN (SFN-GSH), cysteinylglycine conjugate of SFN (SFN-Cys-Gly), cysteine conjugate of SFN (SFN-Cys) and $N$-acetyl cysteine conjugate of SFN (SFN-NAC)

\section{Induction of Apoptosis}

Pro-apoptotic effect of SFN has been documented against prostate cancer cell lines (PC-3), in vitro as well as in xenograft model [16]. Apoptosis by SFN is mediated mainly by caspase-dependent pathways [17-19]. Our own work showed that treatment of prostate cancer cells with SFN generates reactive oxygen species (ROS) with disruption of mitochondrial membrane potential and cytosolic release of cytochrome $c$, resulting in apoptosis in PC-3 and DU145 prostate cancer cells lines [20,21]. In an in vitro experimental model of mouse embryonic fibroblasts, we demonstrated that the B-cell lymphoma 2 (Bcl-2) family of pro-apoptotic proteins (e.g., Bax and Bak) play a critical role in mitochondria-mediated cell death by SFN [22]. This is also accompanied by activation of positive regulators of apoptosis (e.g., Apaf-1) and inhibition of negative regulators (e.g., IAP family of proteins) [22,
23]. As another pro-apoptotic mechanism in prostate cancer cell lines, we also showed SFN-induced inhibition of oncogenic transcription factor STAT3 and reduced levels of STAT3-regulated genes including Bcl-2, cyclinD1, and survivin [24]. Between the various prostate cancer cell lines, our experiments indicated that the androgen-sensitive, p53 wild-type cell line (LNCaP) was relatively more sensitive to SFN-induced apoptosis compared to the androgen-independent, p53 deficient cell lines (PC-3 and DU145) [23].

\section{Inhibition of Cell Cycle Progression}

SFN treatment has been shown to block cell cycle progression at various steps. This has been shown by many groups (including our laboratory) and encompasses a broad range of in vitro and in vivo cancer cell systems. One of the earliest findings was from our laboratory, showing SFN-mediated G2/ 
Fig. 2 Proposed mechanisms of prostate cancer chemoprevention with broccoli-derived sulforaphane

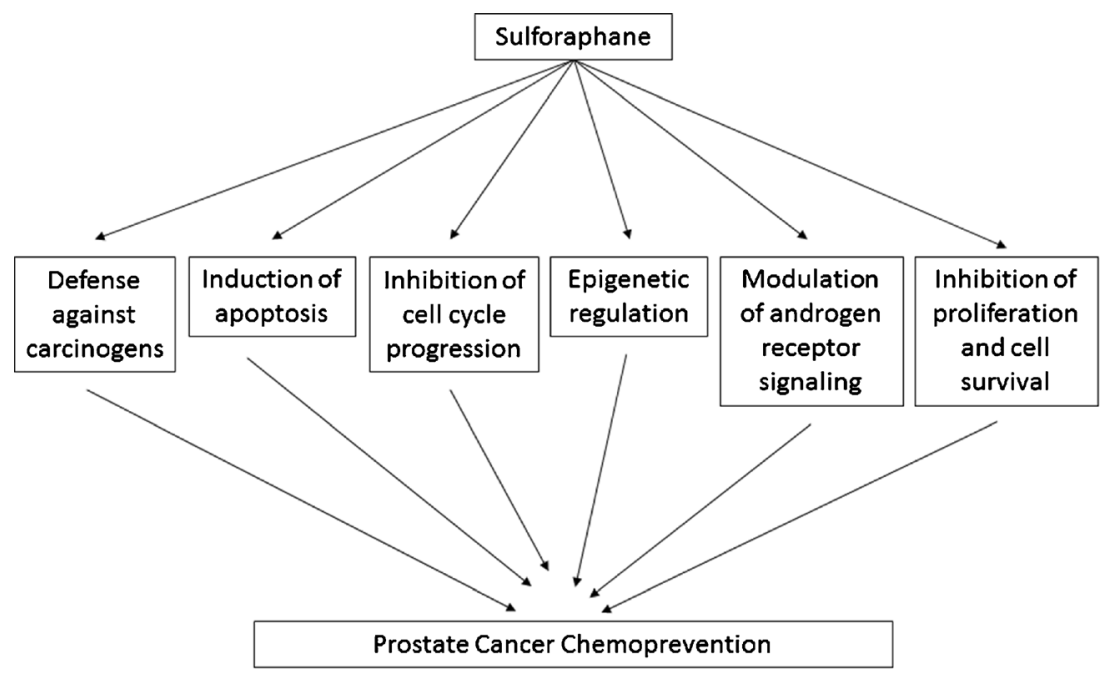

M-phase arrest via inactivation of cyclinB/cyclin-dependent kinase (CDK) 1 complex in PC-3 prostate cancer cells [25]. A similar effect was seen in DU145 cells [26]. Other groups have demonstrated arrest at the G1/S-phase in prostate cancer cells associated with induction of cell cycle regulator $\mathrm{p} 21$, leading to inhibition of cyclinD1/CDK4 and reduced phosphorylation of the retinoblastoma tumor suppressor protein [27-29].

\section{Epigenetic Regulation}

Many dietary factors have been implicated in epigenetic regulation and may be responsible for "dietary chemoprevention". Along the same lines, there has been a greater understanding of epigenetic regulation by SFN. Epigenetic mechanism implicated in progression of prostate cancer includes gene silencing via DNA promoter methylation, histone modification, and changes in miRNA profiles [30]. Clinically, histone deacetylases (HDAC) are known to be highly expressed in prostate cancer and also associated with shorter relapse time after prostatectomy [31]. SFN (and its metabolites) inhibit HDAC and DNA methyltransferases (DNMT) enzymes, facilitating hyperacetylation and hypomethylation of promoter regions of p21 and Bax leading to their re-expression and normal function (i.e., cell cycle arrest and apoptosis). This has been shown in vitro with prostate hyperplasia cell lines (BPH-1) as well as androgen-sensitive and androgen-insensitive prostate cancer cell lines (LNCaP, PC-3) [32]. In another study, SFN treatment reduced the expression of DNMT 1 and 2 and subsequently caused promoter demethylation of cyclinD2 and restoration of its expression thus exerting antiproliferative effects on prostate cancer cells [33]. SFN-rich broccoli modulates epigenetic markers in humans based on a finding of decrease in HDAC activity (and concomitant increase in acetylated histones $\mathrm{H} 3$ and $\mathrm{H} 4$ ) in peripheral blood mononuclear cells a few hours after ingestion of broccoli sprouts in normal healthy volunteers [34]. In addition to competitive enzyme inhibition, SFN lowers the expression of specific HDAC proteins including HDAC3 and HDAC6. Loss of cytoplasmic HDAC6 has important implications in modulating non-histone proteins such as alphatubulin and hsp90, which have roles in controlling cell cycle and androgen receptor (AR) stability, respectively $[35,36]$. Deacetylation of hsp90 by HDAC6 releases AR, allowing it to translocate into the nucleus and modulate gene expression. Recent studies indicate that there may also be a link between the Nrf2 up-regulation hypothesis of chemoprevention and epigenetic modulation by SFN. Inhibition of DNMT1 and 3a by SFN leads to demethylation of $\mathrm{Nrf2}$ promoter, removing its epigenetically silenced status and activation of transcription and protein levels of downstream pathways [37].

\section{Additional Mechanisms of Action}

Prostate cancer is a disease where androgen signaling plays a key role in development and progression of the disease, and this property has been exploited with the currently available therapies. Although SFN is not a potent AR blocker, it has been implicated in the modulation of the receptor expression and function. Our laboratory has shown transcriptional repression of AR in $\mathrm{LNCaP}$ as well as $\mathrm{C} 4-2$ prostate cancer cell lines in vitro [38]. There is also some evidence of epigenetic regulation of AR by SFN via hyperacetylation of the chaperone hsp90, thus destabilizing the receptor and attenuating its signaling [36].

The phosphatidylinositide 3-kinase (PI3K)/AKT/nuclear factor-kappaB (NF- $\mathrm{KB})$ signaling pathway is often enhanced in prostate cancer leading to growth, proliferation, and increased survival of neoplastic cells. Attenuated AKT signaling after treatment with SFN has been shown in prostate cell cultures and also in vivo in a mouse model [transgenic 
adenocarcinoma of mouse prostate (TRAMP)] [39, 40]. In another interesting study by Traka et al., SFN administration to phosphatase and tensin homolog (PTEN)-deleted mouse model for prostate cancer results in complex transcriptional changes which ameliorate the effects of PTEN deletion in the early stages of tumor development [41].

We were the first to show induction of autophagy as a defense mechanism protecting against SFN-induced apoptotic cell death of LNCaP and PC-3 cells in vitro [42]. We also confirmed these findings in vivo using TRAMP model, further showing that inhibition of autophagy by chloroquine increases chemopreventive efficacy of SFN [43].

\section{Chemopreventive Studies in TRAMP Mice}

Oral SFN has been well-studied in TRAMP mouse prostate model with encouraging results. One of the most promising findings is from our laboratory where we showed that SFN inhibits development of PIN and well-differentiated prostate adenocarcinoma [44•]. In our experiments, we randomized 27 TRAMP mice to receive $6 \mu \mathrm{mol}$ SFN orally three times per week or vehicle for SFN (control group). We found that the incidence of PIN and well-differentiated carcinoma were $23-28 \%$ lower in the dorsolateral prostate of SFN-treated TRAMP mice compared with control mice. The area occupied by the well-differentiated carcinoma was also $44 \%$ lower in the SFN-treated mice compared with control mice. Strikingly, SFN was also found to inhibit the incidence and multiplicity of pulmonary metastasis compared to control mice [44•]. The dorsolateral prostate tissue from SFNtreated mice showed decreased cell proliferation as well as increased apoptosis when compared with that from control mice [44•]. Activity of natural killer cells and dendritic cells against TRAMP-C1 target cells was also increased by oral SFN administration [44•]. As discussed above, SFN causes cytoprotective autophagy in prostate cancer cells in culture and in vivo [42, 43]. Thus, it was only logical to hypothesize that cancer chemoprevention by SFN might be enhanced in the presence of an autophagy inhibitor. We tested this hypothesis using chloroquine and demonstrated improved chemopreventive activity of SFN in TRAMP model by co-administration of chloroquine [43]. Keum et al. studied oral administration of broccoli sprouts to TRAMP mice showing significant retardation of prostate tumor growth at doses of $240 \mathrm{mg}$ sprouts/mouse/day administered for 16 weeks [40]. This was also associated with activation of Nrf2/AREsignaling cascade and mitochondria-mediated apoptotic pathway [40]. Moreover, these studies also provided in vivo evidence for cell proliferation inhibition and apoptosis induction by SFN [40, 44•].

\section{Clinical Pharmacokinetics of Sulforaphane}

Given the very promising preclinical efficacy with wellunderstood mechanisms of action, there has been a lot of effort in translating these findings to the clinic to study its efficacy in humans. Initial studies enrolled healthy volunteers aiming to develop analytical techniques for quantification of ITC and its metabolite dithiocarbamates in plasma, serum, erythrocytes, and urine. It was found that a single dose of $200 \mu \mathrm{mol}$ of broccoli sprout (largely SFN) resulted in rapid absorption. ITC concentrations reached peak value of $0.943-2.27 \mu \mathrm{mol} / \mathrm{L}$ in plasma, serum, and erythrocytes at $1 \mathrm{~h}$ after feeding and declined with first-order kinetics (half-life of $1.77 \pm 0.13 \mathrm{~h}$ ). The cumulative excretion at $8 \mathrm{~h}$ was $58.3 \pm 2.8 \%$ of the dose and clearance was $369 \pm 53 \mathrm{~mL} / \mathrm{min}$, indicating active renal tubular secretion [45]. The first formal phase 1 clinical study of a "broccoli sprout extract" was conducted in 2006 [46]. Daily doses ranging from 75 to $300 \mu \mathrm{mol} /$ day of glucosinolate or ITC were administered to 12 healthy volunteers. No major symptomatic toxicities or laboratory parameter abnormalities (including renal, liver, thyroid, and hematological dysfunction) were observed. The study investigators noted very subtle increase in serum alanine transaminase in 11 of 12 patients, but not crossing the upper limit of the normal, and this was attributed to hepatic glycogen storage that occurred with generous meals and relative inactivity during inpatient stay required for the study. One subject had isolated alanine transaminase elevation above normal limits (to a level of $65 \mathrm{IU} / \mathrm{L}$, qualifying as grade 1 toxicity) [46]. There have been many studies since then in healthy adult volunteers as well as in children using various formulations evaluating oral bioavailability and safety of administration [47-50].

Orally administered SFN has good bioavailability in various tissues (including prostate) in animal models $[51,52]$. There are no studies specifically addressing bioavailability of SFN (or its metabolites) in the human prostate tissue, although there are a few studies of "whole broccoli" ingestion by humans and biochemical changes in the prostate. However, bioavailability of SFN in human breast tissue has been evaluated by Cornblatt et al. [53]. In this study, a $200-\mu \mathrm{mol}$ single dose of SFN was orally administered $50 \mathrm{~min}$ prior to surgery to patients undergoing elective reduction mammoplasty. This study found dithiocarbamate concentration of $1.45 \pm 1.12 \mathrm{pmol} / \mathrm{mg}$ in the right breast and $2.00 \pm 1.95 \mathrm{pmol} / \mathrm{mg}$ in the left, approximately $100 \mathrm{~min}$ after ingestion [53]. These levels also correlated with plasma and urine metabolite concentrations and resulted in induction of NQO1 and heme oxygenase- 1 transcripts and enzymatic activity in the mammary tissue [53]. 


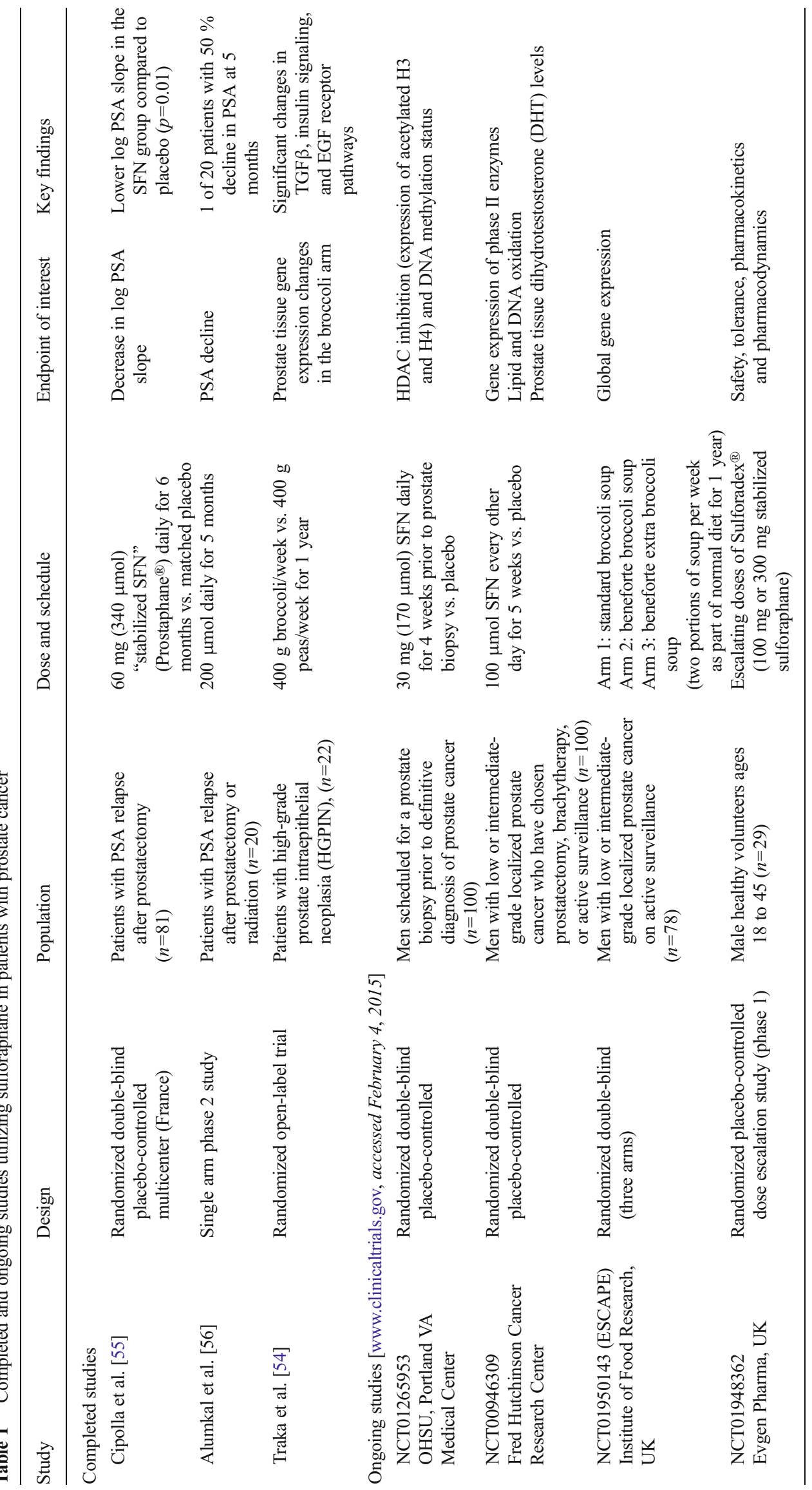




\section{Clinical Trials in Humans Using Sulforaphane}

Completed and ongoing clinical trials on SFN-rich supplements in prostate cancer are summarized in Table 1 (source: www.clinicaltrials.gov, accessed February 4, 2015). These clinical trials are currently in the early phases and have established safety and feasibility of oral administration of broccoli-derived constituents over long periods (up to 1 year) in a wide variety of settings ranging from pre-cancerous prostate conditions (high-grade PIN) to metastatic prostate cancer $[54,55,56 \bullet]$. Two trials recently reported the role of SFN in biochemical [prostate specific antigen (PSA)] recurrence in patients with prostate cancer after definitive surgery or radiation therapy. These trials are summarized below. In a single arm pilot study, Alumkal et al. treated patients with biochemical recurrence of prostate cancer with $200 \mu \mathrm{mol}$ of SFN for up to 20 weeks [56•]. Eighteen of the twenty patients enrolled completed the pre-planned 20 weeks of treatment. There were no grade 3 or 4 toxicities. Seven patients had a decline in PSA with one patient having a decline by $>50 \%$. PSA doubling time improved from 6.1 months pre-study to 9.6 months onstudy [56•]. In another double-blind, multicenter trial from France, 81 prostate cancer patients with biochemical recurrence were randomized to receive $60 \mathrm{mg} \mathrm{SFN}$ or placebo for 6 months. In the intent to treat analysis with 78 patients, the $\log$ PSA slope and PSA doubling time were significantly better in the SFN group (21.9 months) compared to the placebo arm (12.1 months). The SFN group had more gastrointestinal toxicity compared to the placebo but was generally mild [55]. To definitively establish clinical chemoprevention, large resource intensive randomized clinic trials will have to be conducted in high risk populations. Establishment of pharmacodynamic correlates of biological activity will help in efficiently designing such clinical trials. Large phase 3 trials of prostate cancer chemopreventive approaches utilizing selenium, vitamin E, and 5 alpha-reductase inhibitors have not yielded practice-changing results $[57,58]$. Several other dietary agents including soy protein, lycopene, curcumin, green tea (polyphenols), and resveratrol have either failed to show convincing chemopreventive activity in the clinic or are currently in the early phases of clinical development [59, 60, 61•]. We believe that SFN-enriched dietary supplement has great potential as a successful strategy for prostate cancer chemoprevention, and the science is ready for a well-planned transition from the bench to the bedside.

\section{Future Prospects}

To date, only $5 \alpha$ reductase inhibitors, finasteride, and dutasteride have shown a reduced incidence of prostate cancer as chemopreventive agents in two large randomized studies: the Prostate Cancer Prevention Trial (PCPT) and the
Reduction by Dutasteride of Prostate Cancer Events (REDUCE) trial, respectively [58, 62]. In both these studies, subjects who received the $5 \alpha$ reductase inhibitors demonstrated an increased risk of developing high-grade prostate cancer. Given the cost of these agents and increased risk of high-grade prostate cancers, $5 \alpha$ reductase inhibitors are not widely used as chemopreventive agents. SFN, with low toxicity and multiple mechanisms as highlighted in this article, is an attractive chemopreventive agent in prostate and other malignancies. Given the need for extremely large size of chemopreventive trials, it would be more feasible to identify populations at high risk for developing prostate cancer in order to study chemopreventive interventions. Because effects of SFN in chemoprevention were clearly outlined in preclinical mouse models of prostate, it may be reasonable to identify subjects with high-grade PIN to study effects of SFN as a chemopreventive agent in this high-risk population. Additional studies to refine and clearly elucidate mechanisms of SFN activity will help us identify potential prognostic and predictive biomarkers in prostate cancer. These efforts are currently ongoing in our and other laboratories. In summary, broccoli-derived SFN is a promising agent for chemoprevention of prostate cancer based on reproducible bench research findings, and it is ready for translational research at the bedside in the form of welldesigned clinical studies.

Acknowledgments Cited work from the authors' laboratory was funded in part by the US Public Health Service grant RO1 CA115498 which was awarded by the National Cancer Institute.

\section{Compliance with Ethics Guidelines}

Conflict of Interest Ali I. Amjad, Rahul A. Parikh, Leonard J. Appleman, Eun-Ryeong Hahm, Kamayani Singh, and Shivendra V. Singh declare that they have no conflict of interest.

Human and Animal Rights and Informed Consent This article does not contain any studies with human or animal subjects performed by any of the authors.

\section{References}

Papers of particular interest, published recently, have been highlighted as:

- Of importance

- Of major importance

1. Siegel R et al. Cancer statistics, 2014. CA Cancer J Clin. 2014;64(1):9-29.

2. Kolonel LN et al. Vegetables, fruits, legumes and prostate cancer: a multiethnic case-control study. Cancer Epidemiol Biomarkers Prev. 2000;9(8):795-804

3. Cohen JH, Kristal AR, Stanford JL. Fruit and vegetable intakes and prostate cancer risk. J Natl Cancer Inst. 2000;92(1):61-8. 
4.• Liu B et al. Cruciferous vegetables intake and risk of prostate cancer: a meta-analysis. Int J Urol. 2012;19(2):134-41. A metaanlysis of case control and cohort studies combing available epidemiological evidence of prostate cancer chemoprevention with cruciferous vegetables.

5.• Steinbrecher A et al. Dietary glucosinolate intake and risk of prostate cancer in the EPIC-Heidelberg cohort study. Int J Cancer. 2009;125(9):2179-86. A large cohort study from Germany establishing the epidemiological link between dietary glucosinolate intake with reduced risk of prostate cancer. The study uses validated food questionnaires and a national food content database to study the association.

6.• Singh SV, Singh K. Cancer chemoprevention with dietary isothiocyanates mature for clinical translational research. Carcinogenesis. 2012;33(10):1833-42. A comprehensive review by our research team decribing the known in-vitro and in-vivo acitivity of different isothiocyanates against a variety of cancers.

7. Song L, Thornalley PJ. Effect of storage, processing and cooking on glucosinolate content of Brassica vegetables. Food Chem Toxicol. 2007;45(2):216-24.

8. Fahey JW et al. Protection of humans by plant glucosinolates: efficiency of conversion of glucosinolates to isothiocyanates by the gastrointestinal microflora. Cancer Prev Res (Phila). 2012;5(4): 603-11.

9. Fahey JW, Zhang Y, Talalay P. Broccoli sprouts: an exceptionally rich source of inducers of enzymes that protect against chemical carcinogens. Proc Natl Acad Sci U S A. 1997;94(19):10367-72.

10. Zhang Y et al. A major inducer of anticarcinogenic protective enzymes from broccoli: isolation and elucidation of structure. Proc Natl Acad Sci U S A. 1992;89(6):2399-403. Describes one of the pioneer work leading to the identification of isothiocyanates (including sulforaphane) from broccoli and its preliminary chemopreventive efficacy.

11. Fahey JW, Talalay P. Antioxidant functions of sulforaphane: a potent inducer of Phase II detoxication enzymes. Food Chem Toxicol. 1999;37(9-10):973-9.

12. Kensler TW et al. Keap1-nrf2 signaling: a target for cancer prevention by sulforaphane. Top Curr Chem. 2013;329:163-77.

13. Guerrero-Beltran CE et al. Protective effect of sulforaphane against oxidative stress: recent advances. Exp Toxicol Pathol. 2012;64(5): 503-8.

14. Brooks JD, Paton VG, Vidanes G. Potent induction of phase 2 enzymes in human prostate cells by sulforaphane. Cancer Epidemiol Biomarkers Prev. 2001;10(9):949-54.

15. Brooks JD et al. CG island methylation changes near the GSTP1 gene in prostatic intraepithelial neoplasia. Cancer Epidemiol Biomark Prev. 1998;7(6):531-6.

16. Singh AV et al. Sulforaphane induces caspase-mediated apoptosis in cultured PC-3 human prostate cancer cells and retards growth of PC-3 xenografts in vivo. Carcinogenesis. 2004;25(1):83-90.

17. Shankar S, Ganapathy S, Srivastava RK. Sulforaphane enhances the therapeutic potential of TRAIL in prostate cancer orthotopic model through regulation of apoptosis, metastasis, and angiogenesis. Clin Cancer Res. 2008;14(21):6855-66.

18. $\mathrm{Xu} \mathrm{C}$ et al. ERK and JNK signaling pathways are involved in the regulation of activator protein 1 and cell death elicited by three isothiocyanates in human prostate cancer PC-3 cells. Carcinogenesis. 2006;27(3):437-45.

19. Labsch $\mathrm{S}$ et al. Sulforaphane and TRAIL induce a synergistic elimination of advanced prostate cancer stem-like cells. Int J Oncol. 2014;44(5):1470-80.

20. Singh SV et al. Sulforaphane-induced cell death in human prostate cancer cells is initiated by reactive oxygen species. J Biol Chem. 2005;280(20):19911-24.

21. Xiao D et al. Cellular responses to cancer chemopreventive agent $\mathrm{D}, \mathrm{L}$-sulforaphane in human prostate cancer cells are initiated by mitochondrial reactive oxygen species. Pharm Res. 2009;26(7): 1729-38.

22. Choi S, Singh SV. Bax and Bak are required for apoptosis induction by sulforaphane, a cruciferous vegetable-derived cancer chemopreventive agent. Cancer Res. 2005;65(5):2035-43.

23. Choi $\mathrm{S}$ et al. D,L-Sulforaphane-induced cell death in human prostate cancer cells is regulated by inhibitor of apoptosis family proteins and Apaf-1. Carcinogenesis. 2007;28(1):151-62.

24. Hahm ER, Singh SV. Sulforaphane inhibits constitutive and interleukin-6-induced activation of signal transducer and activator of transcription 3 in prostate cancer cells. Cancer Prev Res (Phila). 2010;3(4):484-94.

25. Singh $\mathrm{SV}$ et al. Sulforaphane-induced G2/M phase cell cycle arrest involves checkpoint kinase 2-mediated phosphorylation of cell division cycle 25C. J Biol Chem. 2004;279(24):25813-22.

26. Cho SD et al. Involvement of c-Jun N-terminal kinase in G2/M arrest and caspase-mediated apoptosis induced by sulforaphane in DU145 prostate cancer cells. Nutr Cancer. 2005;52(2):213-24.

27. Wang L et al. Targeting cell cycle machinery as a molecular mechanism of sulforaphane in prostate cancer prevention. Int $\mathrm{J}$ Oncol. 2004;24(1):187-92.

28. Herman-Antosiewicz A et al. Induction of p21 protein protects against sulforaphane-induced mitotic arrest in $\mathrm{LNCaP}$ human prostate cancer cell line. Mol Cancer Ther. 2007;6(5):1673-81.

29. Chiao JW et al. Sulforaphane and its metabolite mediate growth arrest and apoptosis in human prostate cancer cells. Int J Oncol. 2002;20(3):631-6.

30. Dashwood RH, Myzak MC, Ho E. Dietary HDAC inhibitors: time to rethink weak ligands in cancer chemoprevention? Carcinogenesis. 2006;27(2):344-9.

31. Weichert $\mathrm{W}$ et al. Histone deacetylases 1,2 and 3 are highly expressed in prostate cancer and HDAC2 expression is associated with shorter PSA relapse time after radical prostatectomy. Br J Cancer. 2008;98(3):604-10.

32. Myzak $\mathrm{MC}$ et al. Sulforaphane inhibits histone deacetylase activity in $\mathrm{BPH}-1, \mathrm{LnCaP}$ and $\mathrm{PC}-3$ prostate epithelial cells. Carcinogenesis. 2006;27(4):811-9.

33. Hsu A et al. Promoter de-methylation of cyclin D2 by sulforaphane in prostate cancer cells. Clin Epigenetics. 2011;3:3.

34. Myzak MC et al. Sulforaphane retards the growth of human PC-3 xenografts and inhibits HDAC activity in human subjects. Exp Biol Med (Maywood). 2007;232(2):227-34.

35. Clarke JD et al. Differential effects of sulforaphane on histone deacetylases, cell cycle arrest and apoptosis in normal prostate cells versus hyperplastic and cancerous prostate cells. Mol Nutr Food Res. 2011;55(7):999-1009.

36. Gibbs A et al. Sulforaphane destabilizes the androgen receptor in prostate cancer cells by inactivating histone deacetylase 6. Proc Natl Acad Sci U S A. 2009;106(39):16663-8.

37. Zhang $\mathrm{C}$ et al. Sulforaphane enhances Nrf2 expression in prostate cancer TRAMP C1 cells through epigenetic regulation. Biochem Pharmacol. 2013;85(9):1398-404.

38. Kim SH, Singh SV. D,L-Sulforaphane causes transcriptional repression of androgen receptor in human prostate cancer cells. Mol Cancer Ther. 2009;8(7):1946-54.

39. Wiczk A et al. Sulforaphane, a cruciferous vegetable-derived isothiocyanate, inhibits protein synthesis in human prostate cancer cells. Biochim Biophys Acta. 2012;1823(8):1295-305.

40. Keum YS et al. Pharmacokinetics and pharmacodynamics of broccoli sprouts on the suppression of prostate cancer in transgenic adenocarcinoma of mouse prostate (TRAMP) mice: implication of induction of Nrf2, HO-1 and apoptosis and the suppression of Akt-dependent kinase pathway. Pharm Res. 2009;26(10):2324-31.

41. Traka MH et al. The dietary isothiocyanate sulforaphane modulates gene expression and alternative gene splicing in a PTEN null preclinical murine model of prostate cancer. Mol Cancer. 2010;9:189. 
42. Herman-Antosiewicz A, Johnson DE, Singh SV. Sulforaphane causes autophagy to inhibit release of cytochrome $\mathrm{C}$ and apoptosis in human prostate cancer cells. Cancer Res. 2006;66(11):5828-35.

43. Vyas AR et al. Chemoprevention of prostate cancer by d,l-sulforaphane is augmented by pharmacological inhibition of autophagy. Cancer Res. 2013;73(19):5985-95.

44. Singh SV et al. Sulforaphane inhibits prostate carcinogenesis and pulmonary metastasis in TRAMP mice in association with increased cytotoxicity of natural killer cells. Cancer Res. 2009;69(5):2117-25. This article from our group describes the preclinical efficacy of sulforaphane in a transgenic mouse model in delaying prostate cancer progression and metastasis.

45. Ye $\mathrm{L}$ et al. Quantitative determination of dithiocarbamates in human plasma, serum, erythrocytes and urine: pharmacokinetics of broccoli sprout isothiocyanates in humans. Clin Chim Acta. 2002;316(1-2):43-53.

46. Shapiro TA et al. Safety, tolerance, and metabolism of broccoli sprout glucosinolates and isothiocyanates: a clinical phase I study. Nutr Cancer. 2006;55(1):53-62.

47. Egner PA et al. Bioavailability of sulforaphane from two broccoli sprout beverages: results of a short-term, cross-over clinical trial in Qidong, China. Cancer Prev Res (Phila). 2011;4(3):384-95.

48. Cramer JM, Jeffery EH. Sulforaphane absorption and excretion following ingestion of a semi-purified broccoli powder rich in glucoraphanin and broccoli sprouts in healthy men. Nutr Cancer. 2011;63(2):196-201.

49. Egner PA et al. Rapid and sustainable detoxication of airborne pollutants by broccoli sprout beverage: results of a randomized clinical trial in China. Cancer Prevention Research. 2014;7(8):813-23.

50. Singh $\mathrm{K}$ et al. Sulforaphane treatment of autism spectrum disorder (ASD). Proc Natl Acad Sci. 2014;111(43):15550-5.

51. Veeranki OL et al. Organ-specific exposure and response to sulforaphane, a key chemopreventive ingredient in broccoli: implications for cancer prevention. Br J Nutr. 2013;109(1):25-32.

52. Clarke JD et al. Metabolism and tissue distribution of sulforaphane in Nrf2 knockout and wild-type mice. Pharm Res. 2011;28(12): 3171-9.
53. Cornblatt BS et al. Preclinical and clinical evaluation of sulforaphane for chemoprevention in the breast. Carcinogenesis. 2007;28(7): 1485-90.

54. Traka M et al. Broccoli consumption interacts with GSTM1 to perturb oncogenic signalling pathways in the prostate. PLoS One. 2008;3(7):e2568.

55. Cipolla, BG, et al. First double-blind placebo-controlled, multicenter, randomized trial of stabilized natural sulforaphane in men with rising PSA following radical prostatectomy. J Clin Oncol. 2014;32: 5s (Suppl; abstr 5032)

56. Alumkal, JJ, et al. A phase II study of sulforaphane-rich broccoli sprout extracts in men with recurrent prostate cancer. Invest New Drugs. 2014. A key study establishing safety and pharmacodynamic effects of sulforaphane over prolonged period of time in patients with recurrent prostate cancer.

57. Lippman SM et al. Effect of selenium and vitamin $\mathrm{E}$ on risk of prostate cancer and other cancers: the Selenium and Vitamin E Cancer Prevention Trial (SELECT). Jama. 2009;301(1):39-51.

58. Andriole GL et al. Effect of dutasteride on the risk of prostate cancer. N Engl J Med. 2010;362(13):1192-202.

59. Bosland MC et al. Effect of soy protein isolate supplementation on biochemical recurrence of prostate cancer after radical prostatectomy: a randomized trial. JAMA. 2013;310(2):170-8.

60. Sporn MB, Liby KT. Is lycopene an effective agent for preventing prostate cancer? Cancer Prev Res. 2013;6(5):384-6.

61. Thompson Jr IM, Cabang AB, Wargovich MJ. Future directions in the prevention of prostate cancer. Nat Rev Clin Oncol. 2014;11(1): 49-60. A nice review article summarizing preclinical and clinical knowledge on a wide variety of natural and synthetic chemopreventive strategies. Also provides a general guide on approach to future clinical trials.

62. Thompson Jr IM et al. Long-term survival of participants in the Prostate Cancer Prevention Trial. N Engl J Med. 2013;369(7): 603-10. 